\title{
Reakcije oksazola i njihovih derivata u pobuđenom stanju. II. dio: Fotoinducirane inter- $\mathrm{i}$ intramolekulske cikloadicije
}

\author{
I. Šagud* i M. Šindler-Kulyk
}

Zavod za organsku kemiju, Fakultet kemijskog inženjerstva i tehnologije, Sveučilište u Zagrebu,

Marulićev trg 20, 10000 Zagreb, Hrvatska

\begin{abstract}
Sažetak
Oksazolski prsten kao strukturna jedinica u nekom većem sustavu znatno utječe na reakcije u osnovnom i pobuđenom stanju. Reakcijama ciklizacije, cikloadicije i izomerizacije u pobuđenom stanju nastaju nove složene policikličke strukture zanimljive za daljnja biološka istraživanja. Od posebnog interesa su 2,5- i 2,4-disupstituirane kao i 2,4,5-trisupstituirane oksazolske strukture u kojima se kao jedan od supstituenata pojavljuje konjugirani vinil-stirilni sustav. $U$ ovom radu dan je detaljan literaturni pregled inter- i intramolekulskih reakcija oksazolskih derivata u pobuđenom stanju.

Ključne riječi

Oksazol, fotokemija, heterocikli, intermolekulska cikloadicija, intramolekulska cikloadicija, elektrociklizacija
\end{abstract}

\section{Uvod}

Kao što je opisano u prvom dijelu ovog pregleda, ${ }^{* *}$ oksazoli, kao peteročlani heterocikli s dva heteroatoma u prstenu, u pobuđenom stanju skloni su transformacijama prstena, to jest reakcijama fototranspozicije i izomerizacije te nastajanju produkata cijepanjem veza u prstenu. Međutim, ako se oksazolski derivati osvjetljavaju u prisutnosti alkenskih ili karbonilnih spojeva, dolazi do intermolekulskih fotocikloadicijskih reakcija. Ukoliko je oksazolski prsten ugrađen u veći sustav u kojem postoje i drugi kromofori, mijenja se raspodjela energije u pobuđenom stanju i njihova reaktivnost, te dolazi do intramolekulskih reakcija ciklizacije i cikloadicije. Takve reakcije daju pristup novim složenim policikličkim strukturama čiji se opis daje dalje u pregledu.

\section{Fotoinducirane intermolekulske cikloadicije oksazolskih derivata}

Najpoznatije reakcije cikloadicije su svakako termičke [4+2] cikloadicije (Diels-Alder). Jedna od prvih značajnih sinteza na bazi cikloadicije u kojoj sudjeluje oksazolska jezgra je Firestonova sinteza vitamina $B_{6} .{ }^{1}$ Fotoinducirane cikloadicije su važne jer pružaju učinkovit put za konstrukciju složenih policikličkih spojeva do kojih je vrlo zahtjevno, a ponekad i nemoguće doći reakcijama u osnovnom stanju. Od fotoinduciranih intermolekulskih cikloadicija koje uključuju oksazole opisane su [2+2] i [4+4] cikloadicije.

\footnotetext{
*Autor za dopisivanje: dr. sc. Ivana Šagud e-pošta: isagud@fkit.hr

** I. Šagud, M. Šindler-Kulyk, Reakcije oksazola i njihovih derivata u pobuđenom stanju. I. dio: Fototranspozicije u prstenu, Kem. Ind. 67 (1-2) (2017) 39-48, doi: https://doi.org/10.15255/KUI.2017.008. (preceeding paper)
}

\subsection{Fotoinducirane [2+2] cikloadicije oksazolskih derivata}

Reakcija fotoinducirane [2+2] cikloadicije između dvostrukih veza dvaju alkena daje ciklobutane. Paternò-Büchi reakcija (PBR) je reakcija između dvostruke veze karbonila $(\mathrm{C}=\mathrm{O})$ i alkena $(\mathrm{C}=\mathrm{C})$ koja daje oksetane. Grupe Schar$\mathrm{fa}^{2,3}$ i Sekretara ${ }^{4}$ proučavale su 2(3H)-oksazolone i 2,3-dihidrooksazole kao alkenske komponente $\mathrm{u}$ fotocikloadiciji s ketonima i $\alpha$-keto-karboksilatima. Scharf i sur. ${ }^{2}$ su opisali reakciju cikloadicije 3-acetil-2,3-dihidro-2,2-dimetiloksazola s različito supstituiranim karbonilnim spojevima (shema 1).<smiles>[R]C(=O)OC1=CN(C(C)=O)C(C)(C)O1</smiles><smiles>[R]C([R])([R])C1OC(C)(C)N(C(C)=O)C1OC</smiles>

Shema 1 - [2+2] cikloadicija 1 s acetonom, benzofenonom i acetofenonom

Scheme 1 - [2+2] cycloaddition of $\mathbf{1}$ with acetone, benzophenone and acetophenone

Spoj 1 reagira s acetonom i benzofenonom dajući dva regioizomera $\mathbf{2 a}$, b i $\mathbf{3 a}, \mathbf{b}$ u omjeru $2: 7$, odnosno $2: 5$. U 
<smiles>[R]C1O[C@@]2(C)N=C(C)O[C@]1(C)OC2[R]</smiles>
$5 \mathbf{a}: \mathrm{R}=\mathrm{Et}, \mathrm{R}^{\prime}=\mathrm{H}$
b: $\mathrm{R}=\mathrm{Bu}, \mathrm{R}^{\prime}=\mathrm{H}$
c: $R=P h, R^{\prime}=H$
d: $\mathrm{R}=\mathrm{Ph}, \mathrm{R}^{\prime}=\mathrm{CO}_{2} \mathrm{Me}$

4<smiles>[R]C([R])(O)[C@@]([R])(N)[C@@](C)(N)C(C)=O</smiles><smiles>[R]C([R])(O)C(C)(N)C(C)=O</smiles>

Shema 2 - Sinteza eritro- $\beta$-aminoalkohola

Scheme 2 - Synthesis of erythro- $\beta$-amino alcohols

reakciji s acetofenonom nastaje samo spoj 3c. Regioizomeri 3a-c nisu izolirani već su izolirani njihovi derivati s otvorenim oksetanskim prstenom 3'a-c (shema 1).

Slična istraživanja na oksazolskim derivatima nastavlja Griesbeck i sur. ${ }^{5}$ Autori su došli do zaključka da se oksazol može promatrati kao zamaskirani $\alpha$-amino-keton ili $\alpha$-amino-aldehid (slika 1).<smiles>[R]C(=O)C([R])(N)C([R])=O</smiles>

Slika 1 - Oksazol kao zamaskirani $\alpha$-amino-keton/aldehid

Fig. 1 -Oxazole as masked $\alpha$-amino-ketone/aldehyde

Analogno fotocikloadiciji furana s karbonilnim spojevima (foto-aldolni proces) ${ }^{6}$ adicija oksazola i karbonila trebala bi dati maskirane $\alpha$-amino- $\beta$-hidroksi karbonilne spojeve. Opisano je osvjetljavanje 2,4,5-trimetiloksazola (4) s različitim alifatskim i aromatskim aldehidima. U svim slučajevima samo je regioizomer 5a-d izoliran s visokom exo-dijastereoselektivnošću i gotovo kvantitativnim prinosom (shema 2).

Takva regio- i stereoselektivnost oksazolskih derivata u skladu je s fotocikloadicijom furana i karbonila, ali ne i s ponašanjem oksazola u reakcijama kojima su se bavili Sekretar $^{4}$ i Scharf. ${ }^{2,3}$ Dobiveni biciklički oksetani su termički i hidrolitički nestabilni i do otvaranja prstena dolazi već kromatografiranjem na silikagelu ili stajanjem par dana na sobnoj temperaturi u vlažnim otapalima. Oksetane je lako prevesti u $\beta$-amino alkohole reakcijom s trifluoroctenom ili octenom kiselinom. Tako je fotocikloadicija oksazola s karbonilom iz aldehida ili keto-estera dobar način za regio- $\mathrm{i}$ dijastereoselektivnu sintezu eritro- $\beta$-aminoalkohola $(\mathbf{6}, \mathbf{7})$. Nastajanje oksetanskih produkata u reakciji Paternò-Büchi s trimetiloksazolom može se protumačiti mehanizmom u kojem se mora obratiti pažnja na dva faktora. Prvi faktor je geometrija međusustavnog križanja (ISC) koja ovisi o postojanju spin-orbitalnog sprezanja. Drugi faktor je učinak metilne skupine, koji su autori otkrili kod cikličkih monoalkena, gdje metilna skupina kao supstituent na cikloalkenu smanjuje količinu endo-produkta u smjesi u usporedbi s nesupsustituiranim cikloalkenom. Tri projekcije 1,4-biradikala na shemi 3 prikazuju tri reaktivne konformacije (A-C), koje vode u formiranje endo- i exo-produkata ili produkta cijepanja.

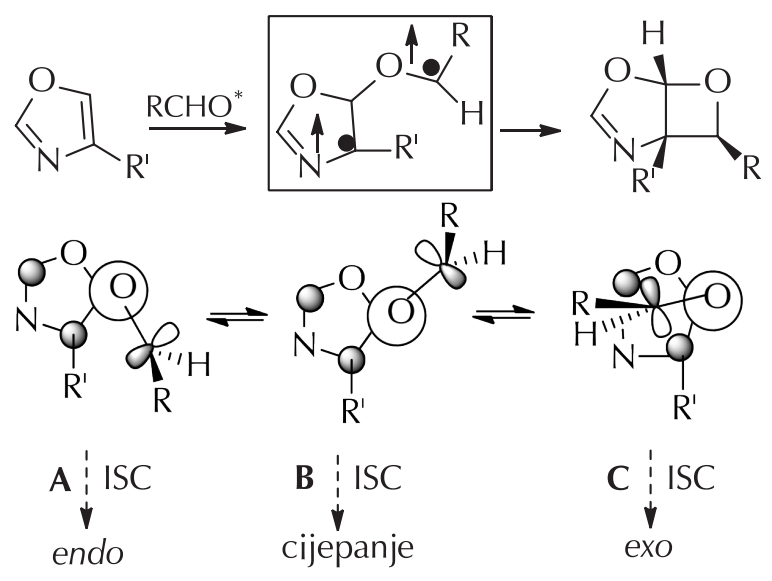

Shema 3 - Geometrije 1,4-biradikala u oksazolu kod fotocikloadicije iz tripletnog stanja

Scheme 3 -1,4-Biradical geometries in oxazole in photocycloaddition from triplet state 
Aldehidi pokazuju sklonost stvaranju veze preko strukture C, pa tako daju exo-oksetane s visokom stereoselektivnošću u PBR. Kod reakcije nesupstituiranih furana i ketoestera $\left(\mathrm{R}=\right.$ alkil, aril; $\left.\mathrm{R}^{\prime}=\mathrm{CO}_{2} \mathrm{R}^{\prime \prime}\right)$ preferirani put je $\mathrm{A}$, te oni u cikloadicijama daju endo-dijastereomere. Ako postoji supstitucija na oksazolu u položaju 4 (R'), onda dodatne steričke smetnje pogoduju stvaranju dijastereomerne smjese, a primjer je adicija metilfenil-glioksilata na trimetiloksazol koja daje 3 : 1 exo/endo smjesu produkata. Autori se u nastavku istraživanja bave ovakvim fotoinduciranim aldolnim reakcijama u svrhu dobivanja $\alpha$-amino- $\beta$-hidroksi karboksilnih kiselina. U slučaju reakcije 2-metil-5-metoksioksazola (8), 7,8 nesupstituiranog u položaju 4, kao diena $\mathrm{s}$ aldehidima detektiran je jedan regioizomerni oksetanski produkt 9a-f u reakcijskoj smjesi (shema 4).

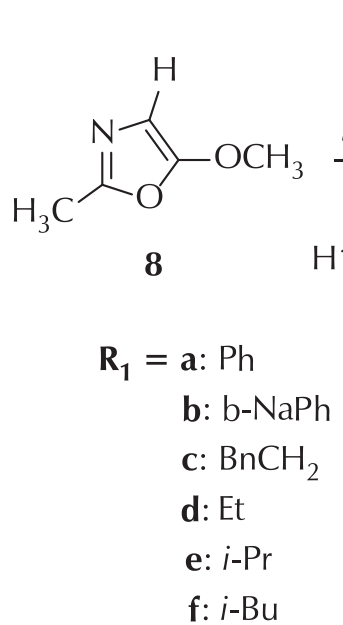<smiles>[R]C1O[C@@]2(OC)O[C@@H]1C2OC</smiles>
9a-f<smiles>[R]C(O)[C@H](NC=[V])C(=O)OC</smiles>

10a-f

Shema 4 - Fotoinducirana adicija oksazola $8 \mathrm{~s}$ aldehidima Scheme 4 - Photoinduced addition of oxazole $\mathbf{8}$ with aldehydes

Primarni fotoprodukti hidrolitički su nestabilni i podliježu otvaranju prstena pri čemu nastaju $\beta$-hidroksi-esteri aminokiselina 10a-f. Autori su svoje istraživanje proširili i na reakcije oksazola supstituiranih u položaju 4 (11a-f) s ben-

zaldehidom. Primarni fotoadukti 12a-f nastaju s visokom dijastereoselektivnošću u svim slučajevima, osim kada su supstituenti u položaju 4 velike skupine (8d-f, shema 5). U slučaju kada je supstituent velika skupina, doprinos konfiguracije A (shema 3) koja vodi u endo-produkte postaje veći. Kromatografiranjem većina produkata 12a-f hidrolizira u $(S, S)$ - $\alpha$-acilamino- $\beta$-hidroksi-estere karboksilne kiseline 13a-f.<smiles>[R]c1nc(C)oc1OC</smiles>

11 a: Me

b: Et

c: $n-\mathrm{Pr}$

d: $i-\operatorname{Pr}$

e: $i-\mathrm{Bu}$

f: $s e c-B u$<smiles>O=Cc1ccccc1</smiles><smiles>[R]C1[C@H]2N=C(C)O[C@]1(OC)O2</smiles>

$12 a-f$

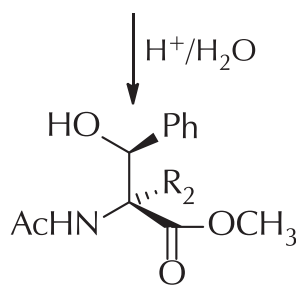

$13 a-f$

Shema 5 - Fotoinducirana adicija oksazola 11a-f s benzaldehidom

Scheme 5 - Photoinduced addition of oxazoles 11a-f and benzaldehyde

Autori su u kasnijem radu ${ }^{9}$ objavili reakcije fotocikloadicije na 2,5-dimetil-4-izobutiloksazolu (14) u svrhu dobivanja $\alpha$-amino- $\beta$-hidroksiketona 15a-e (shema 6). U PBR nastaju samo exo-oksetani 15a-e koji lako hidroliziraju u eritro- $\alpha$-amino- $\beta$-hidroksiketone 16a-e.

U novije vrijeme autori su u istraživanje uključili derivate izoksazola ${ }^{10}$ te ih podvrgnuli reakcijama fotocikloadicije $\mathrm{s}$ alifatskim i aromatskim aldehidima. Došli su do zaključka

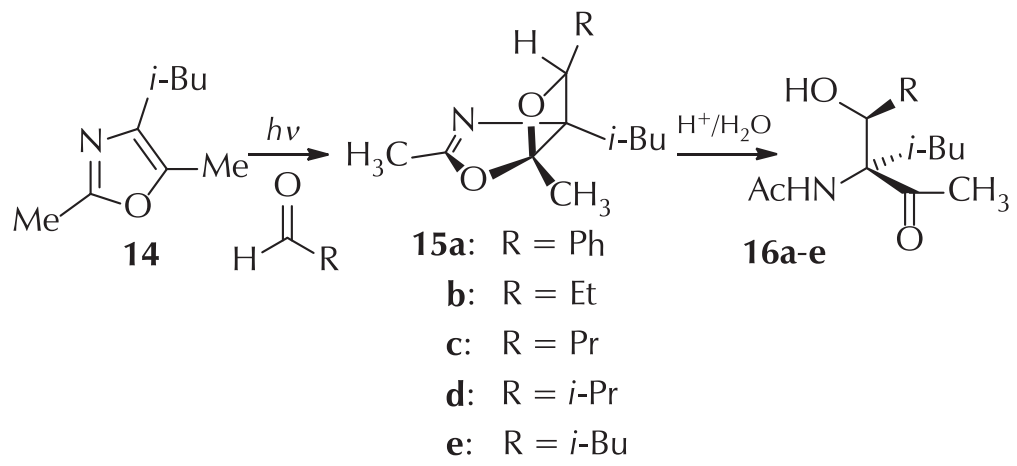

Shema 6 - Paternò-Büchi reakcija oksazola 14 s alifatskim i aromatskim aldehidima

Scheme 6 - Paternò-Büchi reaction of oxazole $\mathbf{1 4}$ with aliphatic and aromatic aldehydes 
da je ta cikloadicija znatno manje učinkovita nego što je to bio slučaj kod oksazola te ostalih heterocikla kojima se bave. Samo kombinacija metiliranih izoksazola i aromatskih aldehida daje adukte koji su dovoljno termički stabilni (shema 7).

$$
\begin{aligned}
\mathbf{1 8} \mathbf{a}: \mathrm{R} & =\mathrm{Ph} \\
\mathbf{b}: \mathrm{R} & =p^{-\mathrm{Tol}} \\
\mathbf{c}: \mathrm{R} & =m-\mathrm{Tol}
\end{aligned}
$$

Shema 7 - Fotocikloadicija izoksazola 17 s aromatskim aldehidima

Scheme 7 -Photocycloaddition of isoxazole $\mathbf{1 7}$ with aromatic aldehydes

\subsection{Fotoinducirane $[4+4]$ cikloadicije oksazolskih derivata}

Dok su [2+2] fotoinducirane cikloadicije uobičajene, one višeg reda su manje poznate. Fotoinducirane [4+4] cikloadicije $^{11}$ uglavnom su otkrivene kod fotodimerizacija naftalena, ${ }^{12}$ antracena, ${ }^{13}$ 2-piridona, ${ }^{14}$ 2-pirona ${ }^{15}$ te kod njihovih fotocikloadicija s dienima. ${ }^{16,17}$ Te cikloadicije događaju se između dva diena i daju osmeročlane karbocikličke spojeve. ${ }^{18,19}$ Ciklički i aciklički $\alpha$-dikarbonilni spojevi reagiraju s alkenima [2+2] i [4+4] fotocikloadicijama. ${ }^{20}$

Xu i sur. ${ }^{21-23}$ objavili su [4+4] cikloadiciju koja uključuje azadiene. Radi se o adiciji o-kinona (1-acetilsatina (IS)) i fenantrenkinona (PQ) s oksazolima (shema 8 i 9). Dolazi do<smiles>O=C1C(=O)c2ccccc2-c2ccccc21</smiles><smiles>[R]</smiles><smiles>[R]c1nc([R])c([Hg])o1</smiles>

$21 \mathrm{a}, \mathrm{b}$

$[4+4]$<smiles>[R]C1=NC2([R])Oc3c(c4ccccc4c4ccccc34)OC2([R])O1</smiles>

$[2+4]$<smiles>[R]C1=NC2([R])OC([R])(O1)OC21C(=O)c2ccccc2-c2ccccc21</smiles>

23b

$[2+2]$

Shema 8 - Fotocikloadicije fenantrenkinona (PQ) s oksazolima 20a i b

Scheme 8 - Photocycloaddition of phenanthrenequinone (PQ) with 20a and $\mathbf{b}$ oxazoles

adicije $\alpha$-dikarbonilnog dijela iz o-kinona $(\mathrm{O}=\mathrm{C}-\mathrm{C}=\mathrm{O})$ i 2-azadienskog dijela iz oksazola $(\mathrm{C}=\mathrm{N}-\mathrm{C}=\mathrm{C})$.

Osvjetljavanje fenantrenkinona (PQ) s 2-metil-4-feniloksazolom 20a daje smjesu produkata [4+4] cikloadicije (21a) i [2+4] cikloadicije (22a). Reakcija 2,4,5-trimetiloksazola 20b s fenantrenkinonom daje produkte [4+4] cikloadicije (21b) i [2+2] cikloadicije (23b) (shema 8). Fotoindu-

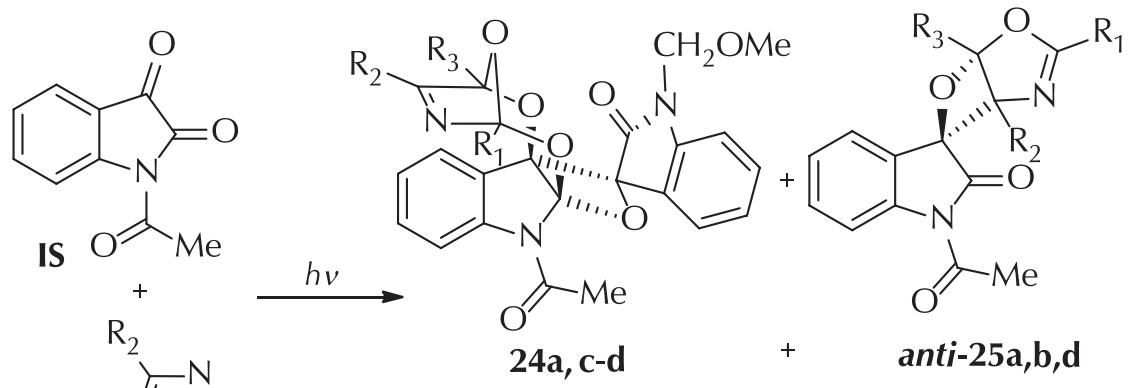

$$
\begin{aligned}
\text { 18a: } R_{1} & =M e, R_{2}=P h, R_{3}=H \\
\text { b: } R_{1} & =R_{3}=R_{2}=M e \\
\text { c: } R_{1} & =R_{3}=H, R_{2}=P h \\
\text { d: } R_{1} & =R_{3}=H, R_{2}=p-C I P h
\end{aligned}
$$

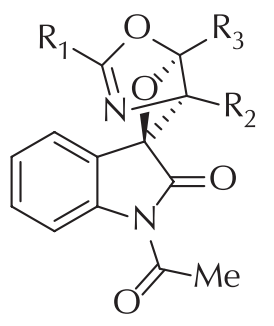

syn-25b,d

Shema 9 - Fotokemijska reakcija 1-acetilsatina (IS) s oksazolima (18a-d)

Scheme 9 - Photochemical reaction of 1-acetyl satin (IS) with oxazoles (18a-d) 


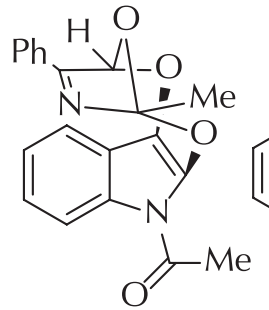

A<smiles>CC(=O)N1C(=O)[C@]2(OC3=N[C@@H]2OC3)c2ccccc21</smiles>

B<smiles>CC(=O)N1C(=O)[C@]2(OC3COC2N3)c2ccccc21</smiles>

C<smiles>CC(=O)n1c2c(c3ccccc31)OC1(C)N=C(c3ccccc3)C(O2)O1</smiles>

D<smiles>CC(=O)n1c([O-])c(O[C@@H]2OC(C)(C)N=C2c2ccccc2)c2ccccc21</smiles>

$\mathbf{E}$<smiles>CC(=O)n1c([O-])c(OC2N=C(c3ccccc3)CO2)c2ccccc21</smiles>

F
G

Slika 2 - Reaktivni međuprodukti

Fig. 2 - Reactive intermediates

ciranom reakcijom 1-acetilsatina (IS) s oksazolom 18a u benzenu nastaju spojevi 24 i 25. Spoj 24a nastaje [4+4] i [2+2] cikloadicijama a produkt 25a nastaje [2+2] cikloadicijom (shema 9). Reakcija IS s 18c daje samo produkt 24c. Reakcija $\mathbf{1 8 b}$ daje dijastereomerne oksetanske produkte anti-25b i syn-25b.

[2+2] fotocikloadicije PQ i IS s oksazolom su regiospecifične, a svi oksetanski produkti imaju strukture acetala. Rezultati mogu biti pojašnjeni mehanizmom preko stabilnog 1,4-biradikalskog međuprodukata u Paternò-Büchi fotocikloadiciji, koji je u ovom slučaju B, a ne $\mathbf{C}$ (slika 2).

[4+4] fotocikloadicije IS s oksazolima su visoko regio- i stereoselektivne te daju kao primarni produkt $\mathbf{A}$ bez njegova regioizomera $\mathbf{D}$ a okazolski prsten nalazi se u syn- položaju s obzirom na 1-acetilsatinski kostur. Da bi objasnili regio- i stereoselektivnost, autori su izračunali standardne entalpije nastajanja svih mogućih biradikalskih međuprodukata pomoću DFT-računa na razini teorije UB3LPY/6-31G*. U E i G IS kostur i oksazolski prsten su gotovo okomiti jedan na drugoga, pa su tako p-orbitale na dva radikalska centra $\mathrm{O}(6)$ i $\mathrm{C}(7)$ gotovo okomite jedna na drugu, što je vrlo pogodno za brzo međusustavno križanje (ISC). Nakon ISC-a u G slijedi cijepanje veze $\mathrm{C}-\mathrm{O}$, pri čemu se biradikal zbog velike udaljenosti između radikala vraća u reaktante (IS i oksazol). Za razliku od toga nakon ISC-a u E, kod kojega postoji mala udaljenost između radikala, slijedi rotacija p-orbitale prema unutra na atomu $C(7)$, što rezultira stvaranjem veze i nastankom [4+4] adukta A. Taj adukt ima eksperimentalno potvrđenu sin-konfiguraciju. Rotacija p-orbitale na atomu C(7) potpomognuta je sekundarnom interakcijom između orbitale fenila na $\mathrm{C}(5)$ te p sustava u IS. $U$ toj konformaciji biradikal $\mathbf{E}$ stabilniji je od regioizomernog 1,6-diradikala F. Autori su detaljno opisali novi tip [4+4] cikloadicije između dva heterodiena te dali objašnjenje za visoku regio- i stereoselektivnost. Istraživanje nastavljaju na takvim adicijama i u kasnijem radu, ${ }^{23}$ gdje opisuju i putove adicije izokinolin-1,3,4-triona na oksazole. U slučaju izokinolin-1,3,4-triona dolazi samo do [2+2]-fotocikloadicije na supstituirane oksazole i formiranja policikličkih spirookestanskih struktura (slika 3).<smiles>[R]C1=N[C@@H]2C([R2])[C@@]3(O[C@@]2(OC)O1)c1ccccc1C(=O)N3[X]</smiles><smiles>[R]C1=N[C@]2([R2])[C@]3(OC)O[C@]2(C(=O)N([X])C3=O)c2ccccc2C1=O</smiles>

Slika 3 - Spirooksetanski produkti Fig. 3 - Spirooxetane products 


\section{Fotoinducirane intramolekulske ciklizacije i cikloadicije oksazolskih derivata}

Oksazolske derivate koji u položaju 2, 4 ili 5 imaju stirilne ili analogne heterocikličke skupine u konjugaciji (slika $4^{*}$ ) studira grupa Šindler i sur. ${ }^{32-37}$ nastavljajući fotokemiju sličnih furanskih, ${ }^{24,27,29}$ tiofenskih, ${ }^{28,31}$ pirolskih, ${ }^{25,26}$ i sidnonskih $^{30}$ heksatrienskih sustava (slika 5).<smiles>Cc1nc(I)c(S)o1</smiles><smiles>[R][X]c1ccc(/C=C/C)cc1</smiles><smiles>[X][X]1(O)ccccc1=O</smiles>

Slika 4 - Ispitivani oksazolski spojevi Fig. 4 - Investigated oxazole compounds

Cilj istraživanja je fotokemijska sinteza hetero-policikličkih spojeva, strukturnih osnova za biološka ispitivanja. Studirani heksatrienski sustavi podliježu reakcijama cis-trans izomerizacije, ciklizacije i cikloadicije.

* Crvene točke prikazuju spojna mjesta vezivanja supstituenata na oksazolski prsten.

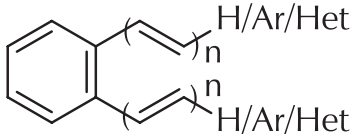

$\mathrm{n}=0,1,2$<smiles>[X]=CN</smiles><smiles>[X]c1ccccc1</smiles>

Slika 5 - Ispitivani spojevi

Fig. 5 - Investigated compounds

\subsection{Fotoinducirane izomerizacije i ciklizacije}

Proučavane su fotokemijske reakcije izomerizacije i ciklizacije okazolskih derivata ${ }^{32-35}$ koji u položaju 5 na prstenu imaju stirilni (26) ili heteroaril-etenilni supstituent (27-32). Tako su po prvi put sintetizirani fuzionirani oksazolski derivati (33-39) primjenjujući energiju svjetla (shema 10).

Rađena su i foto-fizikalna mjerenja, gdje su praćeni procesi koji se događaju u sustavu u kratkim vremenima nakon pobude. ${ }^{33-35}$ Stirilni derivati oksazola u položaju 5 pokazali su u blagim uvjetima i kratkim vremenima pobude ponašanje analogno stilbenu. Teorijski računi ravnoteže između konformera trans-izomera podudaraju se s eksperimentalnim mjerenjima i pokazale su da je ravnoteža znatno pomaknuta prema s-trans-26 rotameru (shema 11). ${ }^{33}$

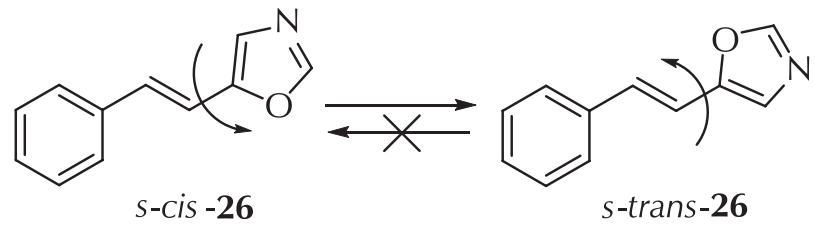

Shema 11 - Konformacijski izomeri trans-5-stiriloksazola Scheme 11 - Conformational isomers of trans-5-styryloxazole

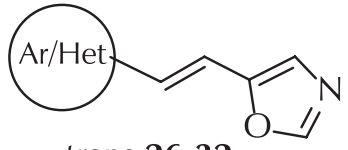

trans 26-32<smiles>C=CCCCC=Cc1cnco1</smiles>

cis 26-32

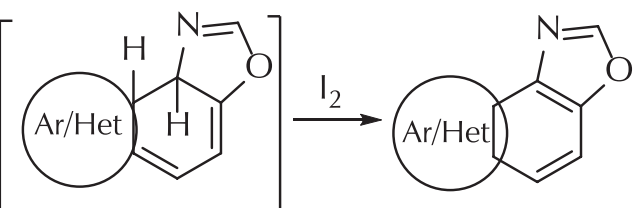

$\mathrm{DH}$
33-39

Het/Ar = aril, 2-furil, 2-tienil,

3-tienil, 2-, 3-, 4-piridil

Shema 10 - Sinteza naftooksazola te furo-, tieno- i piridinobenzoksazola

Scheme 10 - Synthesis of naphthoxazoles and furo-, thieno- and pyridinobenzoxazoles 


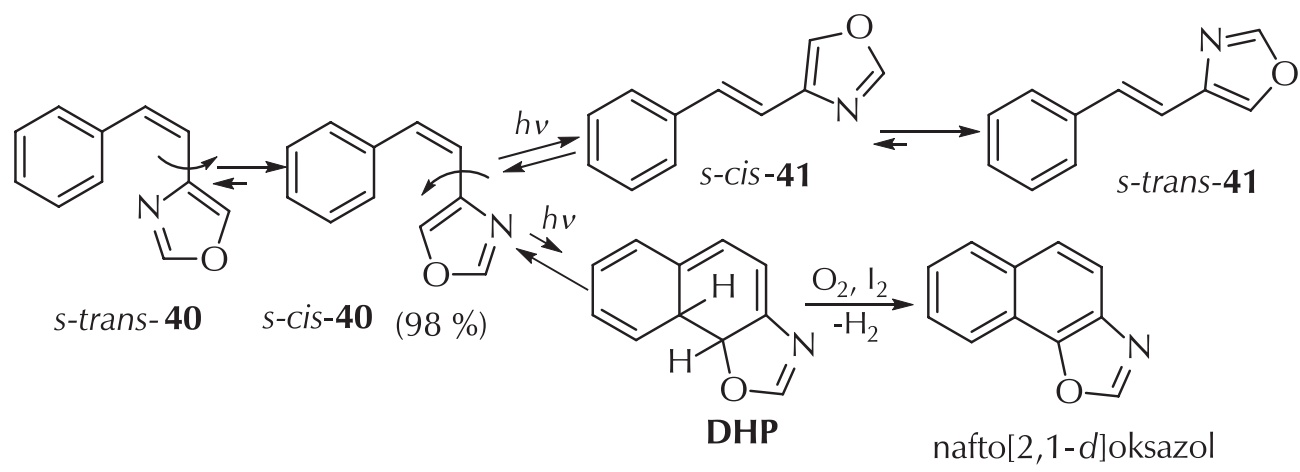

Shema 12 - Ponašanje 4-stiriloksazola u pobuđenom stanju

Scheme 12 - Excited state behaviour of 4-styryloxazole

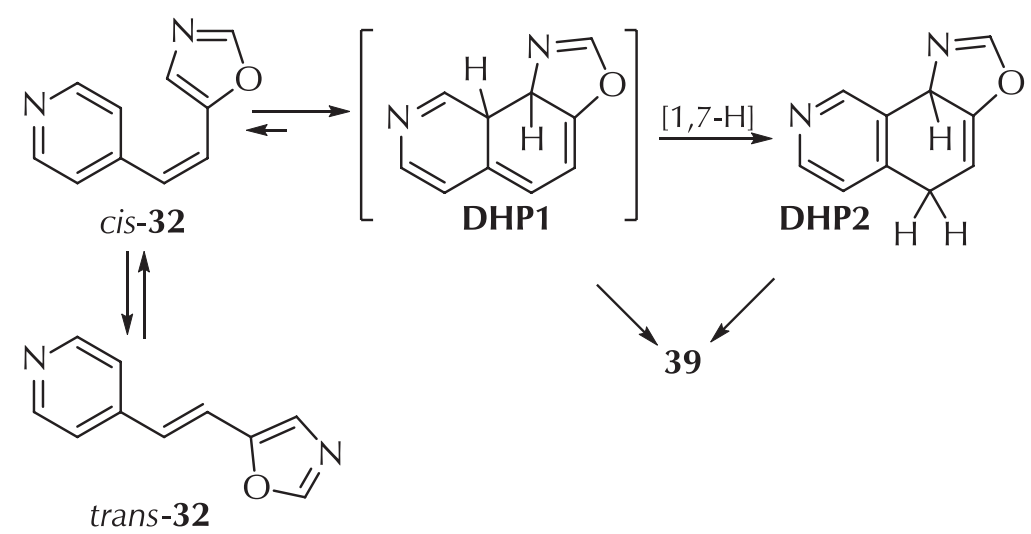

Shema 13 - Ponašanje 5-(2-(piridin-4-il)vinil)oksazola u pobuđenom stanju Scheme 13 - Excited state behaviour of 5-(2-(pyridin-4-yl)vinyl)oxazole derivative

Kod cis-izomera situacija je drugačija jer obje kompetitivne reakcije (reakcija izomerizacije i reakcija ciklizacije) karakterizira niži prinos nego kod stilbena. Taj učinak pripisuje se smanjenoj gustoći elektronskog oblaka koju uzrokuje prisutnost $\mathrm{N}$ atoma. $\mathrm{U}$ nastavku su autori studirali i ponašanje 2- i 4-stiril-oksazolskih derivata. ${ }^{34}$ Različiti položaj stirilne skupine s obzirom na heteroatome (kisik i dušik) u oksazolu utječe na raspodjelu naboja u molekuli što dalje ima utjecaj na kompetitivne procese relaksacije. U shemi $12 \mathrm{u}$ nastavku prikazani su procesi nakon pobude 4-stiriloksazola. Utvrđeno je da jedan konformer prevladava i kod cis- i trans-izomera (shema 12).

U slučaju trans-2-stiriloksazola oba konformera su prisutna u podjednakim količinama, a kod cis-izomera većina je u obliku s-cis-rotamera. Zaključak je da u kratkim vremenima pobude prvo dolazi do rotacije oko jednostruke veze, gdje se jasno vidi da su određeni rotameri preferirani te nakon nekog vremena dolazi do izomerizacije, gdje je fotostacionarno stanje pomaknuto prema cis-izomeru. Dužim osvjetljavanjem uz prisutnost kisika i joda uz izomerizacije dolazi i do ciklizacije kod 4- i 5-stiriloksazola (shema 10), dok 2-stiriloksazol ne ciklizira. ${ }^{34}$
Također su studirani 5-stirilioksazolski derivati u kojima je benzenska jezgra zamijenjena piridinskom u položaju 2 (30) odnosno 3 (31) ili 4 (32). ${ }^{55}$ Očekivalo se da će doći do pojave solvatokromizma i u emisijskim i apsorpcijskim spektrima, ali do toga nije došlo. Izostanak se objašnjava nepostojanjem međusustavnog prijelaza (ICT) zbog premale razlike između donorskog i akceptorskog karaktera dviju heteroaromatskih skupina. Osvjetljavanjem 4-piridinskog derivata (32) u uvjetima bez kisika "ulovljen" je i međuprodukt DHP2 (shema 13).

Prinosi u reakcijama ciklizacije svih piridiletenil-oksazolskih derivata bili su niži od onih kod 5-stiriloksazolskih derivata.

\subsection{Fotoinducirane cikloadicije 4- $\mathrm{i}$ 5-vinilstirilnih derivata oksazola}

Sintetizirani derivati 4-(2-vinilstiril)oksazol (42) i 5-(2-vinilstiril)oksazol (43) u pobuđenom stanju daju primarno fuzi- 
onirane oksazolinske produkte $\mathbf{4 4}$ odnosno $\mathbf{4 7}$ te u tragovima oksazolski produkt $\mathbf{4 5} .{ }^{36}$ Oksazolinski derivati 44 i 47 su relativno nestabilni i spontano prelaze u esterski derivat 46 odnosno formamidni derivat 48 (shema 14).

$\mathrm{Na}$ taj način dobiveni modificirani biciklički produkti pogodni su kao međuprodukti u daljnjim totalnim sintezama potencijalno biološki aktivnih supstancija.

\subsection{Fotoinducirane cikloadicije 2-vinilstirilnih derivata oksazola}

Ponašanje u pobuđenom stanju 2-(2-vinilfenil)etenil-oksazola razlikuje se od prethodno istraživanih hetero-stilbenskih sustava kao i od ponašanja prethodno opisanih 4- i 5-derivata oksazola. Fotokemijskom pobudom ti derivati daju kao većinske produkte okso-premoštene kinoline $(\mathbf{5 0 b}, \mathbf{c})$ formalnom intramolekularnom [4+2] fotocikloadicijom odnosno 10p, 6p elektrociklizacijom (shema 15). ${ }^{37}$

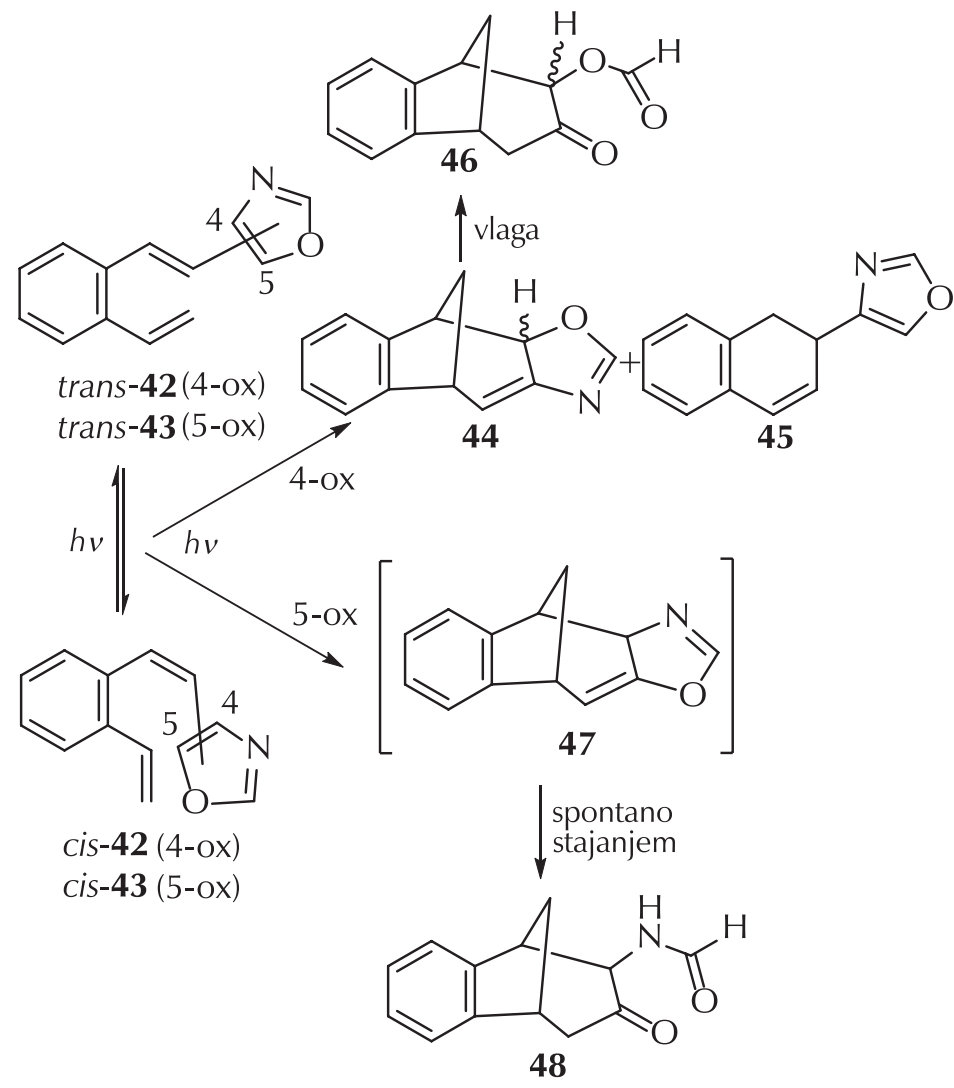

Shema 14 - Osvjetljavanje spojeva $\mathbf{4 2}$ i 43

Scheme $\mathbf{1 4}$ - Irradiation of compounds $\mathbf{4 2}$ and $\mathbf{4 3}$

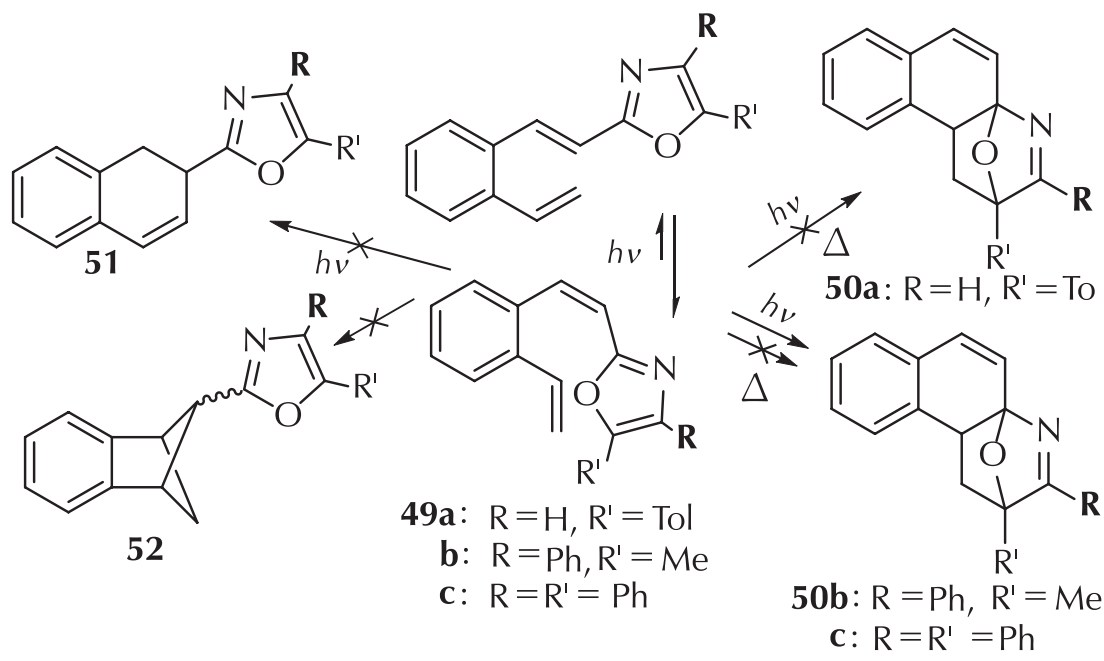

Shema 15 - Fotokemijska reaktivnost derivata 49a-c

Scheme 15 - Photochemical reactivity of compounds 49a-c 
TD-DFT računi ${ }^{37}$ pokazali su dobro slaganje s eksperimentalnim rezultatima te su potvrdili da najveći utjecaj na samu reakciju u pobuđenom stanju ima fenilni supstituent u položaju 4 na oksazolskom prstenu. Takvi okso-premošteni derivati lako se mogu prevesti u kinolinske derivate, te je time dobiven novi put ka benzo[f]kinolinima.

\section{Popis kratica \\ List of abbreviations}

$\begin{aligned} \text { ICT } & \text { - intramolekulski prijenos naboja } \\ & \text { - intramolecular charge transfer } \\ \text { IS } & -1 \text {-acetilsatin } \\ & \text { - 1-acetyl satin } \\ \text { ISC } & \text { - geometrija međusustavnog križanja } \\ & \text { - intersystem crossing } \\ \text { PBR } & \text { - Paternò-Büchi reakcija } \\ & \text { - Paternò-Büchi reaction } \\ \text { PQ } & \text { - fenantrenkinon } \\ & \text { - phenanthrenequinone }\end{aligned}$

\section{Literatura}

\section{References}

1. R. A. Firestone, E. E. Harris, W. Reuter, Synthesis of pyridozine by Diels-Alder reactions with 4-methyl-5-alkoxy oxazoles, Tetrahedron 23 (1967) 943-955, doi: https://doi. org/10.1016/0040-4020(67)85043-9.

2. M. Weuthen, H-D. Scharf, J. Runsink, 3-Acetyl-2,3-dihydro-2,2-dimethyloxazol; Darstellung, Eigenschaften und Einsatz als olefinischer Partner in der Paternò-Büchi-Reaktion, Chem. Ber. 120 (1987) 1023-1026, doi: https://doi. org/10.1002/cber.19871200623.

3. M. Weuthen, H.-D. Scharf, J. Runsink, R. Vaßen, Chirale Induktion bei photochemischen Reaktionen, VIII. Diastereoselektive Photo-Aldol-Reaktion von chiralen $\alpha$-Ketoestern mit 2,3-Dihydrooxazolen, Chem. Ber. 121 (1988) 971-976, doi: https://doi.org/10.1002/cber.19881210523.

4. S. Sekretár, J. Kopecký, A. Martvoň, Photochemical reactions of substituted 2(3H)-oxazolones with carbonyl compounds, Collect. Czech. Chem. Commun. 47 (1982) 1848-1855, doi: https://doi.org/10.1135/cccc19821848.

5. A. G. Griesback, M. Giege, J. Lex, Oxazole-Carbonyl photocycloadditions: Selectivity pattern and synthetic route to erythro $\alpha$-amino, $\beta$-hydroxy ketones, Chem. Commun. 7 (2000) 589-590, doi: https://doi.org/10.1039/B000578I.

6. A. G. Griesbeck, S. Buhr, M. Fiege, H. Schmickler, J. Lex, Stereoselectivity of Triplet Photocycloadditions: Diene-Carbonyl Reactions and Solvent Effects, J. Org. Chem. 63 (1998) 3847-3854, doi: https://doi.org/10.1021/jo971767l.

7. A. G. Griesback, S. Bondock, Photo aldol reactions with 5-methoxyoxazoles: Highly regio- and diastereoselective synthesis of $\alpha$-amino- $\beta$-hydroxy carboxylic acid derivatives, Can. J. Chem. 81 (2003) 555-559, doi: https://doi. org/10.1139/v03-029.

8. A. G. Griesbeck, S. Bondock, Photocycloaddition of 5-Methoxyoxazoles to Aldehydes and $\alpha$-Keto Esters: A Comprehensive View on Stereoselectivity, Triplet Biradical Conformations, and Synthetic Applications of Paternò-Büchi Adducts, Aust. J. Chem. 61 (2008) 573-580, doi: https://doi. org/10.1071/CH08168.
9. S. Bondock, A. G. Griesback, Diastereoselective Photochemical Synthesis of $\alpha$-Amino- $\beta$-hydroxyketones by Photocycloaddition of Carbonyl Compounds to 2,5-Dimethyl-4-isobutyloxazole, Monatsh. Chem. 137 (2006) 765-777, doi: https://doi.org/10.1007/s00706-006-0474-4.

10. A. G. Griesbeck, M. Franke, J. Neudörfl, H. Kotaka, Photocycloaddition of aromatic and aliphatic aldehydes to isoxazoles: Cycloaddition reactivity and stability studies, Beilstein J. Org. Chem. 7 (2011) 127-134, doi: https://doi.org/10.3762/ bjoc.7.18.

11. G. Kaupp, $[4+4]$-Cycloaddition Reactions in the Total Synthesis of Naturally Occurring Eight-Membered Ring Compounds, Angew. Chem., Int. Ed. 31 (1992) 422-424, doi: https://doi.org/10.1002/anie.199204221.

12. S. Kohmoto, T. Kobayashi, J. Minami, X. Ying, K. Yamaguchi, T. Karatsu, A. Kitamura, K. Kishikawa, M. Yamamoto, Trapping of 1,8-Biradical Intermediates by Molecular Oxygen in Photocycloaddition of Naphthyl-N-(naphthylcarbonyl)carboxamides; Formation of Novel 1,8-Epidioxides and Evidence of Stepwise Aromatic Cycloaddition, J. Org. Chem. 66 (2001) 66-73, doi: https://doi.org/10.1021/jo0010227.

13. H. Bouas-Laurent, A. Castellan, J.-P. Desvergne, R. Lapouyade, Photodimerization of anthracenes in fluid solutions: (part 2) mechanistic aspects of the photocycloaddition and of the photochemical and thermal cleavage, Chem. Soc. Rev. 30 (2001) 248-263, doi: https://doi.org/10.1039/B006013P.

14. S. McN. Sieburth, u M. Harmata (ur.), Advances in Cycloaddition, Vol. 5, Greenwich CT, 1998., 85-118.

15. F. G. West, u M. Lautens (ur.), Advances in Cycloaddition, Vol. 4, Greenwich CT, 1997., 1-40.

16. A. Albini, E. Fasani, F. Giavarini, Photochemical reaction between naphthalenecarbonitriles and dienes, J. Org. Chem. 53 (1988) 5601-5607, doi: https://doi.org/10.1021/ jo00259a002.

17. G. McSkimming, J. H. R. Tucker, H. Bouas-Laurent, J.-P. Desvergne, An Anthracene-Based Photochromic System That Responds to Two Chemical Inputs, Angew. Chem., Int. Ed. 39 (2000) 2167-2169, doi: https://doi.org/10.1002/15213773(20000616)39:12<2167::AID-ANIE2167>3.0. $\mathrm{CO} ; 2-9$.

18. F. Muller, J. Mattay, Photocycloadditions: control by energy and electron transfer, Chem. Rev. 93 (1993) 99-117, doi: https://doi.org/10.1021/cr00017a006.

19. D. I. Schuster, G. Lem, N. A. Kaprinidis, New insights into an old mechanism: [2 + 2] photocycloaddition of enones to alkenes, Chem. Rev. 93 (1993) 3-22, doi: https://doi. org/10.1021/cr00017a001.

20. G. Jones II., Synthetic application of the Paternò-Büchi reaction u A. Padwa (ur.), Organic Photochemistry, Vol. 5, Marcel Dekker, New York, 1981., str. 1-122.

21. Y. Zhang, L. Wang, M. Zhang, H.-K. Fun, J.-H. Xu, Photoinduced [4 + 4] Cycloadditions of o-Quinones with Oxazoles, Org. Lett. 26 (6) (2004) 4893-4895, doi: https://doi. org/10.1021/ol048028t.

22. L. Wang, Y.-C. Huang, Y. Liu, H.-K. Fun, Y. Zhang, J.-H. Xu, Photoinduced [4 + 4], [4 +2], and [2+2] Cycloadditions of o-Quinones with Oxazoles: Chemo-, Regio-, and Diastereoselectivity, J. Org. Chem. 75 (2010) 7757-7768, doi: https:// doi.org/10.1021/jo101764f.

23. C.-M. Huang, H. Jing, R.-Z. Wang, C. K. Quah, H.-K. Fun, Y. Zhang, Photocycloadditions of substituted oxazoles with isoquinoline-1,3,4-trione chemo-, regio-, diastereoselectivities and transformation of the photocycloadducts, Org. Biomol. Chem. 11 (2013) 5023-5033, doi: https://doi.org/10.1039/ C3OB40645H. 
24. M. Šindler-Kulyk, L. Špoljarić, Ž. Marinić, Photochemistry of beta-(2-Furyl)Substituted $O$-Divinylbenzenes, Heterocycles 29 (1989) 679-682, doi: https://doi.org/10.3987/COM-894629.

25. M. Šindler-Kulyk, N. Basarić, Photochemical Approach to Hetero Polycyclic Compounds, Kem. Ind. 51 (2002) 169175.

26. N. Basarić, Ž. Marinić, M. Šindler-Kulyk, Photochemical Formation of Novel Pyrrolo[3, 2-b]-6, 7-benzobicyclo[3.2.1] octa-2, 6-diene, J. Org. Chem. 68 (2003) 7524-7527, doi: https://doi.org/10.1021/jo0346454.

27. I. Škorić, M. Šindler-Kulyk, Svjetlom do heteropolicikličkih spojeva, Kem. Ind. 56 (2007) 135-143.

28. D. Vidaković, I. Škorić, M. Horvat, Ž. Marinić, M. Šindler-Kulyk, Photobehaviour of 2- and 3-heteroaryl substituted o-divinylbenzenes; formation of fused 2,3- and 3,2-heteroareno-benzobicyclo[3.2.1] octadienes and 3-heteroaryl benzobicyclo[2.1.1] hexenes, Tetrahedron 64 (2008) 39283934, doi: https://doi.org/10.1016/j.tet.2008.02.062.

29. I. Škorić, M. Šmehil, Ž. Marinić, K. Molčanov, B. Kojić-Prodić, M. Šindler-Kulyk, Photochemistry of $\omega$-(o-vinylphenyl)- $\omega^{\prime}$-(phenyl/2-furyl) butadienes: New approach to 4-substituted benzobicyclo[3.2.1]octadienes, J. Photochem. Photobiol. A: Chemistry 207 (2009) 190-196, doi: https:// doi.org/10.1016/j.jphotochem.2009.07.008.

30. K. Butković, D. Vuk, Ž. Marinić, J. Penić, M. Šindler-Kulyk, Synthesis and photochemistry of 3-(o-stilbeneyl)-4-H/Me/ Ph-sydnones; intramolecular cyclization to 1,2-benzodiazepines and/or quinolines, Tetrahedron 66 (2010) 9356-9362, doi: https://doi.org/10.1016/j.tet.2010.10.013.

31. D. Vuk, Ž. Marinić, K. Molčanov, B. Kojić-Prodić, M. Šindler-Kulyk, Photochemical transformation of $\beta, \beta^{\prime}$-dithienyl substituted o-divinylbenzenes leading to 1,2-dihydronaphthalenes or fused pentacyclic compounds: first evidence of electrocyclization process via 2,3-dihydronaphthalene intermediates, Tetrahedron 68 (2012) 6873-6880, doi: https:// doi.org/10.1016/j.tet.2012.06.019.

32. I. Šagud, F. Faraguna, Ž. Marinić, M. Šindler-Kulyk, Photochemical approach to naphthoxazoles and fused hetero-benzoxazoles from 5-(phenyl/heteroarylethenyl)oxazoles, J. Org. Chem. 76 (2011) 2904-2908, doi: https://doi. org/10.1021/jo1025942.

33. I. Šagud, M. Šindler-Kulyk, A. Spalletti, U. Mazzucato, Photoisomerization and Photocyclization of 5-Styryloxazole, Croat. Chem. Acta 87 (2014) 327-333, doi: https://doi. org/10.5562/cca2443.

34. V. Botti, F. Elisei, U. Mazzucato, I. Šagud, M. Šindler-Kulyk, A. Spalletti, Effect of the positional isomerism on the photoreactivity of styryloxazole, J. Photochem. Photobiol. A: Chemistry 316 (2016) 95-103, doi: https://doi.org/10.1016/j. jphotochem.2015.10.022.

35. V. Botti, F. Elisei, F. Faraguna, Ž. Marinić, U. Mazzucato, I. Šagud, M. Šindler-Kulyk, A. Spalletti, Deactivating effect of the pyridine $n, \pi^{*}$ states on the photoreactivity of 5-[2-(pyrid$-n$-yl)ethenyl]oxazole ( $n=2,3$ and 4), J. Photochem. Photobiol. A: Chemistry 329 (2016) 262-272, doi: https://doi. org/10.1016/j.jphotochem.2016.07.009.

36. I. Šagud, S. Božić, Ž. Marinić, M. Šindler-Kulyk, Photochemical approach to functionalized benzobicyclo[3.2.1]octene structures via fused oxazoline derivatives from 4- and 5-(o-vinylstyryl)oxazoles, Beilstein J. Org. Chem. 10 (2014) 2222-2229, doi: https://doi.org/10.3762/bjoc.10.230.

37. I. Šagud, I. Antol, Ž. Marinić, M. Šindler-Kulyk, Photoinduced Intramolecular formal [4+2] Cycloaddition of Aryl-Substituted o-Vinylstyryl-2-oxazoles To Form Benzo[f]quinoline Derivatives: Experimental Results and Theoretical Interpretation, J. Org. Chem. 80 (2015) 9535-9541, doi: https://doi. org/10.1021/acs.joc.5b01504.

\section{SUMMARY \\ Excited State Reactions of Oxazoles and Their Derivatives. Part II: Photoinduced Inter- and Intramolecular Cycloadditions

\author{
Ivana Šagud ${ }^{*}$ and Marija Šindler-Kulyk
}

The oxazole ring in a complex system has a substantial impact on the reactions in ground and excited state. By cyclisation, cycloaddition and isomerization reactions in the excited state new complex polycyclic structures are formed that are interesting for further investigation. Of particular interest are 2,5-, 2,4-disubstituted and 2,4,5-trisubstituted oxazole structures in which one of the substituents is a conjugated vinyl-styryl segment. In this paper a detailed literature review is given into photoinduced inter- and intramolecular reactions of oxazole derivatives.

\section{Keywords}

Oxazole, photochemistry, heterocycles, intermolecular cycloaddition, intramolecular cycloaddition, electrocyclisation

Faculty of Chemical Engineering and Technology

Review

University of Zagreb, Marulićev trg 20

10000 Zagreb, Croatia 